\title{
$\mathrm{AC}$ 와 DC 노이즈가 있는 적분기 시스템에서 측정에러의 영향을 감소시키는 스위칭 상태 궤환 제어기의 설계 및 분석
}

\section{Design and Analysis of a Switching State Feedback Controller to Reduce the Measurement Error Effect for a Chain of Integrators System under AC and DC Noise}

\author{
오 상 영, 최 호 림* \\ (Sang-Young $\mathrm{Oh}^{1}$ and Ho-Lim Choi ${ }^{1,{ }^{*}}$ ) \\ ${ }^{1}$ Department of Electrical Engineering, Dong-A University
}

\begin{abstract}
In this paper, we propose a controller capable of reducing the effect of measurement errors under AC and DC noise. Typically, the control system measures data through a sensor. If sensor noise is included in a controller via the feedback channel, the signal is distorted and the entire system cannot work normally. Therefore, some appropriate action to counter the measurement error effect is essential in the controller design. Our controller is equipped with a gain-scaling factor and a compensator to reduce the effect of measurement error in the feedback signal. Also, we use a switching control strategy to enhance the performance of the controller regarding convergence speed. Our proposed controller can therefore effectively reduce the AC and DC noise of the sensor. We analyze the proposed controller by Laplace transform technique and our control method is verified via MATLAB simulation.
\end{abstract}

Keywords: chain of integrators, measurement error, regulation, gain-scaling factor, switching control

\section{I. 서론}

일반적으로 제어 시스템은 센서를 통해 데이터들을 측정 하고 시스템이 동작하게 된다. 이러한 데이터를 측정 하는데 있어서 센서 잡음이 포함되면 궤환(feedback) 신호를 왜곡시 키고 이로 인하여 제어 시스템이 정상적으로 작동할 수 없게 만든다[3,5,8]. 따라서 이러한 측정 노이즈에 대한 영향을 최 소화 하기 위해 센서 잡음이 포함되어 있는 데이터들을 분석 처리하는 적절한 조치가 필수적이다[2,4,9]. 이미 적분기 시스 템에서 $\mathrm{AC}$ 측정 노이즈를 감소시키는 많은 연구가 있었다 $[7,11,12]$. 기존의 논문 $[7,11,12]$ 에서는 $\mathrm{AC}$ 측정 노이즈만 고 려한 적분기 시스템을 다루었다. 하지만 우리의 경우는 $\mathrm{AC}$ 측정 노이즈 뿐만 아니라 $\mathrm{DC}$ 측정 노이즈가 함께 포함된 경 우의 적분기 시스템을 다루었고 추가적으로 스위칭 제어 기 법을 혼용하여 제어기의 수렴속도를 개선하였다.

본 논문에서는 적분기 시스템에 대해서 출력신호인 상태 변수 $x_{1}(t)$ 에 측정 노이즈가 포함되었을 경우 이득조절 요소 (gain-scaling factor)가 추가된 제어기를 설계하였고, $x_{1}(t)$ 를 제외한 상태변수 $x_{i}(t)$ 에 측정 노이즈가 포함되었을 경우 보 상기(compensator)를 추가하여 제어기를 설계하였다. 그리고 $\mathrm{AC}$ 측정 노이즈는 미지의 정현파 함수로, $\mathrm{DC}$ 측정 노이즈는 알 수 없는 상수 값으로 나타남을 가정하고 $n$ 차의 적분기 시스템에 제안된 제어기를 적용하였다. 따라서 제어기의 입 력에 이득조절 요소와 보상기가 추가된 상태 궤환 제어기는

\footnotetext{
* Corresponding Author

Manuscript received October 2, 2013 / revised October 28, 2013 / accepted November 21, 2013

오상영, 최호림: 동아대학교 전기공학과

(rkdeh99@hanmail.net/hlchoi@dau.ac.kr)

※ 본 논문은 동아대학교 학술연구비 지원에 의하여 연구되었음.
}

출력 $y(t)$ 의 최종 수렴범위(ultimate bound)의 크기를 최소화 하였다. 또한, 이득 조절요소인 $\gamma$ 값과 보상기 상수인 $\beta$ 값 이 커짐에 따라 늦어지는 수렴속도(convergence speed)를 줄이 기 위하여 스위칭 제어 기법(switching control technique)을 적 용하여 수렴속도를 줄이는 방법을 적용했다[7,10]. 제안된 제 어기가 인가된 전체 적분기 시스템을 라플라스 변환 기법으 로 분석을 하였고 MATLAB 시뮬레이션을 통해 제어기의 성 능을 확인하여 시스템의 유효성을 검증하였다.

\section{II. 시스템 구성 및 문제의 정의}

기존에 $[4,7,9,11,12]$ 와 같이 $n$ 차 적분기 시스템을 다룬 많 은 연구가 있었다. $n$ 차 적분기 시스템은 다음과 같다.

$$
\begin{gathered}
\dot{x}_{1}(t)=x_{2}(t) \\
\dot{x}_{2}(t)=x_{3}(t) \\
\vdots \\
\dot{x}_{n}(t)=u(t) \\
y(t)=x_{1}(t)
\end{gathered}
$$

식 (1)을 행렬로 나타내면 다음과 같다.

$$
\begin{gathered}
\dot{x}(t)=A x(t)+B u(t) \\
y(t)=C x(t)
\end{gathered}
$$

여기서 $x(t)=\left[x_{1}(t), \cdots, x_{n}(t)\right]^{T} \in R^{n}$ 은 상태, $u(t) \in R$ 는 입력, $y(t) \in R$ 는 출력, $(A, B)$ 는 Brunovsky canonical form으로 $A=\left[a_{i j}\right] \in R^{n \times n}\left(j=i+1\right.$ 일 때 $a_{i j}=1$, 그 외의 경우는 $a_{i j}=$ $0), B=[0, \cdots, 0,1]^{T} \in R^{n \times 1}, C=[1,0, \cdots, 0] \in R^{1 \times n}$ 이다. 이 시스 템에서 상태 궤환 제어기는 다음과 같다[1]. 


$$
u(t)=K x(t)
$$

$K=\left[k_{1}, \cdots, k_{n}\right]$ 으로 정의한다. 식 (2)로부터 식 (4)를 대입 하여 정리하면 다음과 같은 폐루프 시스템(closed-loop system) 으로 표현할 수 있다.

$$
\dot{x}(t)=(A+B K) x(t)
$$

여기서 $A+B K$ 가 Hurwitz이기 위한 $K$ 의 값을 선택하면 $x(t) \rightarrow 0$ 이 되어 전체 폐루프 시스템은 안정하게 된다. 하지 만 이 시스템에서 센서의 오작동으로 인하여 상태변수 $x_{i}(t)$ 에 측정 노이즈가 포함되는 것을 가정하면 다음과 같다.

$$
\bar{x}_{i}(t)=x_{i}(t)+d_{i}(t), \quad(1 \leq i \leq n)
$$

$\bar{x}_{i}(t)$ 는 측정 노이즈가 포함된 상태변수이고 $d_{i}(t)$ 는 측정 노이즈이다. 측정 노이즈가 포함된 폐루프 시스템으로 표현 하면 아래와 같이 나타낼 수 있다.

$$
\begin{aligned}
\dot{x}(t) & =A x(t)+B K \bar{x}(t) \\
& =A x(t)+B K(x(t)+d(t)) \\
& =(A+B K) x(t)+B K d(t)
\end{aligned}
$$

식 (7)에서 $d(t)=\left[d_{1}(t), \cdots, d_{i}(t)\right]^{T}$ 의 영향으로 $x$ 의 모든 성분의 최종 수렴범위가 커지게 된다. 그리고 이득 $K$ 값과 연동되어 $\mathrm{AC}$ 와 $\mathrm{DC}$ 측정 노이즈가 포함된 상태 궤환 제어 기는 측정 노이즈의 영향을 제어하는데 어려움이 있음을 알 수 가있다. 따라서 이러한 문제를 해결하기 위하여 III 장에 서 $x_{1}(t)$ 에 측정 노이즈가 포함되었을 때는 제어기의 이득에 이득조절 요소가 추가된 상태 궤환 제어기를, IV 장에서는 $x_{1}(t)$ 을 제외한 $x_{i}(t)$ 에 측정 노이즈가 포함되었을 때는 보 상기가 추가된 제어기를 제안한다. 그리고 이득조절 요소와 보상기의 영향으로 늦어지는 수렴속도를 줄이기 위해 스위 칭 제어 기법[3,10]을 혼용하여 적용하는 방법을 $\mathrm{V}$ 장에서 제안하고, $\mathrm{VI}$ 장에서 제안된 제어기가 인가된 시스템을 시뮬 레이션 하여 유효성을 검증하였다.

Assumption 1: $n$ 차 시스템에서 상태변수 $x_{i}(t)$ 에 포함되 는 측정 노이즈를 다음과 같은 $\mathrm{AC}$ 와 $\mathrm{DC}$ 성분으로 나타남 을 가정한다.

$$
d_{i}(t)=d_{i}+\alpha_{i} \sin \omega_{i} t
$$

여기서 $d_{i} \geq 0$ 는 $\mathrm{DC}$ 측정 노이즈의 크기, $\alpha_{i} \geq 0$ 는 $\mathrm{AC}$ 측 정 노이즈의 크기, $\omega_{i} \geq 1$ 는 $\mathrm{AC}$ 측정 노이즈의 주파수이다. 그리고 $d_{i}, \alpha_{i}, \omega_{i}$ 는 알 수 없는 임의의 값으로 가정한다.

Remark 1: 일반적으로 $\mathrm{AC}$ 측정 노이즈는 정현파 함수에 대한 사례를 주로 가지고 있다[2,6,9]. $\mathrm{AC}$ 측정 노이즈는 미지 의 정현파 함수로, $\mathrm{DC}$ 측정 노이즈는 임의의 상수 값으로 나 타남을 가정하였다. 기존의 논문 [2,9]에서는 알고 있는 외부 시스템으로부터 발생하는 정현파 $\mathrm{AC}$ 측정 노이즈를 가정하 여 에러의 크기와 주파수를 알 수 있음을 가정한 반면, 우리 의 경우는 미지의 $\alpha_{i}, \omega_{i}$ 를 가정하고 추가적으로 $\mathrm{DC}$ 측정
노이즈로 인해 발생하는 알 수 없는 $d_{i}$ 를 더하여 노이즈에 대한 조건을 더 일반화시켰다.

\section{III. $x_{1}(t)$ 에 측정 노이즈가 포함된 $n$ 차 상태 궤환 제어기}

$n$ 차 적분기 시스템에서 $x_{2}(t), \cdots, x_{n}(t)$ 는 정상적인 궤환 신호를 가지고 $x_{1}(t)$ 의 궤환 신호에 $\mathrm{AC}$ 와 $\mathrm{DC}$ 측정 노이즈 가 발생함을 가정한다. 이러한 시스템을 제어하기 위해 이득 조절 요소가 추가된 제어기를 식 (9)와 같이 제안한다.

$$
\begin{aligned}
u(t)= & \frac{k_{1}}{\gamma^{n}}\left\{\int_{y(0)}^{t}\left(\frac{d}{d \theta} y(\theta)\right) d \theta\right\} \\
& +\frac{k_{2}}{\gamma^{n-1}} x_{2}(t)+\frac{k_{3}}{\gamma^{n-2}} x_{3}(t)+\cdots+\frac{k_{n}}{\gamma} x_{n}(t)
\end{aligned}
$$

노이즈가 포함된 상태변수 $x_{1}(t)$ 은 $y(t)=\left(x_{1}(t)+d_{1}+\right.$ $\left.\alpha_{1} \sin \omega_{1} t\right)$ 와 같이 표현할 수 있다. $y(0)$ 는 출력 $y(t)$ 의 초 기값으로 미리 알고 있다고 가정한다. $y(0)$ 는 출력 $y(t)$ 의 초기값으로 시스템을 운영하기 전에 알 수 있다. 예를 들어 볼-빔 시스템, 자기부상 시스템에서 $y(0)$ 는 초기 볼의 위치 이다. 따라서 시스템을 구동하기 전에 알 수 있고 기타 많은 시스템에서 출력은 위치를 나타내고 있어 시스템을 구동하 기 전에 알 수 있기 때문에 $y(0)$ 의 값을 알고 있다고 가정 하였다. 제안된 제어기를 적용하여 폐루프 시스템으로 표현 하면 식 (10)과 같다.

$$
\begin{aligned}
\dot{x}_{1}(t)= & x_{2}(t) \\
\dot{x}_{2}(t)= & x_{3}(t) \\
& \vdots \\
\dot{x}_{n}(t)= & \frac{k_{1}}{\gamma^{n}}\left\{\int_{y(0)}^{t}\left(\frac{d}{d \theta} y(\theta)\right) d \theta\right\} \\
& +\frac{k_{2}}{\gamma^{n-1}} x_{2}(t)+\frac{k_{3}}{\gamma^{n-2}} x_{3}(t)+\cdots+\frac{k_{n}}{\gamma} x_{n}(t) \\
y(t)= & x_{1}(t)
\end{aligned}
$$

응답을 확인하기 위해 라플라스 변환을 적용하면 다음과 같다.

$$
\begin{aligned}
s X_{1}(s)-x_{1}(0) & =X_{2}(s) \\
s X_{2}(s)-x_{2}(0) & =X_{3}(s) \\
& \vdots \\
s X_{n}(s)-x_{n}(0)= & \frac{k_{1}}{\gamma^{n}}\left(X_{1}(s)+\frac{\alpha_{1} \omega_{1}}{s^{2}+\omega_{1}^{2}}\right) \\
& +\frac{k_{2}}{\gamma^{n-1}} X_{2}(s)+\frac{k_{3}}{\gamma^{n-2}} X_{3}(s)+\cdots+\frac{k_{n}}{\gamma} X_{n}(s)
\end{aligned}
$$

여기서 $k_{n}<0, \gamma>0$ 일 때, 극점의 음수항은 최종치 정리 (final value theorem)를 통해 0 으로 수렴하고 최종치 정리를 할 수 없는 아래의 항이 남게 된다.

$$
X_{1}(s)=\frac{k_{1} \alpha_{1} \omega_{1}}{\left(s^{2}+\omega_{1}^{2}\right)\left(\gamma^{n} s^{n}-k_{n} \gamma^{n-1} s^{n-1}-k_{n-1} \gamma^{n-2} s^{n-2}-\cdots-k_{1}\right)}
$$

출력을 확인하기 위해 계산하면, 


$$
\begin{gathered}
X_{1}(s)=\frac{P s+Q}{s^{2}+\omega_{1}^{2}} \\
x_{1}(t)=P \cos \omega_{1} t+\frac{Q}{\omega_{1}} \sin \omega_{1} t \\
y(t)=\sqrt{P^{2}+\left(\frac{Q}{\omega_{1}}\right)^{2}} \sin \left(\omega_{1} t+\theta\right)
\end{gathered}
$$

여기서 $P, Q$ 는 아래와 같은 식으로 표현할 수 있다.

$$
P=\frac{k_{1} \alpha_{1} \omega_{1} b}{a^{2}+\omega_{1}^{2} b^{2}}, \quad Q=-\frac{k_{1} \alpha_{1} \omega_{1} a}{a^{2}+\omega_{1}^{2} b^{2}}
$$

그리고 $P, Q$ 에서 표현된 $a, b$ 는 다음과 같은 식으로 각각 표 현할 수 있다.

- 홀수 차수 일 때

$$
\text { if } \begin{aligned}
n= & (3,7,11, \cdots) \\
a= & -\omega_{1}^{n-1} k_{n} \gamma^{n-1}+\omega_{1}^{n-3} k_{n-2} \gamma^{n-3}-\omega_{1}^{n-5} k_{n-4} \gamma^{n-5}+\cdots+k_{1} \\
b= & \omega_{1}^{n-1} \gamma^{n}+\omega_{1}^{n-3} k_{n-1} \gamma^{n-2}-\omega_{1}^{n-5} k_{n-3} \gamma^{n-4}+\omega_{1}^{n-7} k_{n-5} \gamma^{n-6} \\
& -\cdots+k_{2} \gamma
\end{aligned}
$$

else $n=(5,9,13, \cdots)$

$$
\begin{aligned}
a= & \omega_{1}^{n-1} k_{n} \gamma^{n-1}-\omega_{1}^{n-3} k_{n-2} \gamma^{n-3}+\omega_{1}^{n-5} k_{n-4} \gamma^{n-5}-\cdots+k_{1} \\
b= & -\omega_{1}^{n-1} \gamma^{n}-\omega_{1}^{n-3} k_{n-1} \gamma^{n-2}+\omega_{1}^{n-5} k_{n-3} \gamma^{n-4}-\omega_{1}^{n-7} k_{n-5} \gamma^{n-6} \\
& +\cdots+k_{2} \gamma
\end{aligned}
$$

- 짝수 차수 일 때

if $n=(2,6,10, \cdots)$

$$
\begin{aligned}
a & =\omega_{1}^{n} \gamma^{n}+\omega_{1}^{n-2} k_{n-1} \gamma^{n-2}-\omega_{1}^{n-4} k_{n-3} \gamma^{n-4}+\cdots+k_{1} \\
b & =\omega_{1}^{n-2} k_{n} \gamma^{n-1}-\omega_{1}^{n-4} k_{n-2} \gamma^{n-3}+\omega_{1}^{n-6} k_{n-4} \gamma^{n-5}-\cdots+k_{2} \gamma \\
\text { if } n & =(4,8,12, \cdots) \\
a & =-\omega_{1}^{n} \gamma^{n}-\omega_{1}^{n-2} k_{n-1} \gamma^{n-2}+\omega_{1}^{n-4} k_{n-3} \gamma^{n-4}-\cdots+k_{1} \\
b & =-\omega_{1}^{n-2} k_{n} \gamma^{n-1}+\omega_{1}^{n-4} k_{n-2} \gamma^{n-3}-\omega_{1}^{n-6} k_{n-4} \gamma^{n-5}+\cdots+k_{2} \gamma
\end{aligned}
$$

따라서 이득조절 요소가 추가된 $n$ 차 상태 궤환 제어기의 최종 출력 식은 아래와 같다.

$$
\begin{gathered}
y(t)=\sqrt{P^{2}+\left(\frac{Q}{\omega_{1}}\right)^{2}} \sin \left(\omega_{1} t+\theta\right) \\
=\frac{k_{1} \alpha_{1}}{\sqrt{a^{2}+\omega_{1}^{2} b^{2}}} \sin \left(\omega_{1} t+\theta\right) \\
y(t) \leq \frac{k_{1} \alpha_{1}}{\gamma^{\frac{n}{2}} \Delta} \sin \left(\omega_{1} t+\theta\right)
\end{gathered}
$$

$\Delta$ 는 최종 수렴범위의 크기에 영향을 끼치는 $\gamma$ 를 제외한 $\omega, k$ 의 상수 계수이다. 식 (22)에서 이득조절 요소인 $\gamma$ 값이 클수록 0 에 가까운 출력이 나오게 되어 최종 수렴범위의 크 기를 줄일 수 있다.

\section{IV. $x_{i}(t)$ 에 측정 노이즈가 포함된 $n$ 차 상태 궤환 제어기}

$n$ 차 적분기 시스템에서 $x_{1}(t), \cdots, x_{i-1}(t), x_{i+1}(t), \cdots, x_{n}(t)$ 는 정상적인 궤환 신호를 가지고 $x_{i}(t)$ 의 궤환 신호에 $\mathrm{AC}$ 와
$\mathrm{DC}$ 측정 노이즈가 발생함을 가정한다. 측정 노이즈를 감소 시키기 위해 보상기 $\left(\frac{1}{\beta}\left(\delta(t)-\frac{1}{\beta} e^{-\frac{1}{\beta} t}\right)\right)$ 가 포함된 제어기를 식 (23)과 같이 제안한다.

$$
\begin{aligned}
u(t)= & k_{1} x_{1}(t)+k_{2} x_{2}(t)+\cdots+k_{i-1} x_{i-1}(t) \\
& +k_{i}\left(x_{i}(t)+d_{i}+\alpha_{i} \sin \omega_{i} t\right) * \frac{1}{\beta}\left(\delta(t)-\frac{1}{\beta} e^{-\frac{1}{\beta} t}\right) \\
& +k_{i+1} x_{i+1}(t)+\cdots+k_{n} x_{n}(t)
\end{aligned}
$$

여기서 $x_{i}(t)$ 는 $x_{1}(t)$ 을 제외한 상태변수 $\left(x_{i}(t), 2 \leq i \leq n\right)$, * 는 컨벌루션 심볼, 보상기 상수 $\beta \geq 1$ 이다. 제안된 제어기 를 적용하여 폐루프 시스템으로 표현하면 식 (24)와 같다.

$$
\begin{aligned}
\dot{x}_{1}(t)= & x_{2}(t) \\
\dot{x}_{2}(t)= & x_{3}(t) \\
& \vdots \\
\dot{x}_{n}(t)= & k_{1} x_{1}(t)+k_{2} x_{2}(t)+\cdots+k_{i-1} x_{i-1}(t) \\
& +k_{i}\left(x_{i}(t)+d_{i}+\alpha_{i} \sin \omega_{i} t\right) * \frac{1}{\beta}\left(\delta(t)-\frac{1}{\beta} e^{-\frac{1}{\beta} t}\right) \\
& +k_{i+1} x_{i+1}(t)+\cdots+k_{n} x_{n}(t) \\
y(t)= & x_{1}(t)
\end{aligned}
$$

응답을 확인하기 위해 라플라스 변환을 적용하면 다음과 같다.

$$
\begin{aligned}
s X_{1}(s)-x_{1}(0)= & X_{2}(s) \\
s X_{2}(s)-x_{2}(0)= & X_{3}(s) \\
\vdots & \\
s X_{n}(s)-x_{n}(0)= & k_{1} X_{1}(s)+k_{2} X_{2}(s)+\cdots+k_{i-1} X_{i-1}(s) \\
& +k_{i}\left(X_{i}(s)+\frac{d_{i}}{s}+\frac{\alpha_{i} \omega_{i}}{s^{2}+\omega_{i}^{2}}\right)\left(\frac{s}{\beta s+1}\right) \\
& +k_{i+1} X_{i+1}(s)+\cdots+k_{n} X_{n}(s)
\end{aligned}
$$

여기서 $k_{n}<0, \beta>0$ 일 때, 극점의 음수항은 최종치 정리를 통해 0 으로 수렴하고 최종치 정리를 할 수 없는 아래의 항이 남게 된다.

$$
\begin{aligned}
& X_{1}(s)= \\
& \frac{k_{i} \alpha_{i} \omega_{i} s}{\left(s^{2}+\omega_{i}^{2}\right)\left(\beta s^{n+1}+\left(1-k_{n} \beta\right) s^{n}-\left(k_{n}+k_{n-1} \beta\right) s^{n-1}-\cdots-k_{1} \beta s-k_{1}\right)}
\end{aligned}
$$

출력을 확인하기 위해 계산하면,

$$
\mathrm{y}(\mathrm{t}) \leq \frac{k_{i} \alpha_{i} \omega_{i}}{\beta^{0.33} \nabla} \sin \left(\omega_{i} t+\theta\right)
$$

$\nabla$ 는 최종 수렴범위의 크기에 영향을 끼치는 $\beta$ 를 제외한 $\omega, k$ 의 상수 계수이다. 식 (27)에서 보상기 상수인 $\beta$ 값이 클수록 0 에 가까운 출력이 나오게 되어 최종 수렴범위의 크 기를 줄일 수 있다.

Remark 2: 적분기 시스템에서 모든 상태변수 $x_{i}(t)$ 에 측 정 노이즈가 포함된 경우도 있지만 특정 상태변수에만 노이 즈가 포함된 경우 우리의 제안된 제어기를 유용하게 적용할 
수 있다. 예를 들어 3차 적분기 시스템에서 $x_{2}(t)$ 와 $x_{3}(t)$ 에 측정 노이즈가 포함되어 있다면 제안된 방식을 확장시켜 제 어기를 설계할 수 있다. 제안된 제어기를 적용하면 출력은

$$
\mathrm{y}(\mathrm{t}) \leq \frac{k_{2} \alpha_{2} \omega_{2}}{\beta^{0.33} \Delta} \sin \left(\omega_{2} t+\theta_{2}\right)+\frac{k_{3} \alpha_{3} \omega_{3}}{\beta^{0.33} \nabla} \sin \left(\omega_{3} t+\theta_{3}\right)
$$

와 같이 표현이 된다. 여기서 $\Delta, \nabla$ 는 최종 수렴범위의 크기 에 영항을 끼치는 $\beta$ 를 제외한 $\omega, k$ 의 상수 계수이다. 따라 서 보상기 상수 $\beta$ 값을 증가 시켜 측정 노이즈를 감소 시킬 수 있다.

\section{V. 스위칭 제어 기법}

\section{1. $\gamma, \beta$ 와 수렴속도의 관계}

이득 조절요소가 추가된 $n$ 차 상태 궤환 제어기에서 최종 수렴범위의 크기와 수렴속도를 결정하는 라플라스 식은 아 래와 같다.

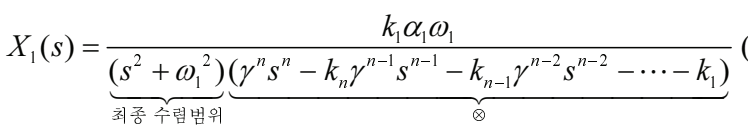

$\otimes$ 은 수렴속도를 결정한다. 수렴속도와 관련된 식을 인수분 해 하여 정리하면 식 (29)와 같다.

$$
\otimes=\left(\gamma s+\sigma_{1}\right)\left(\gamma s+\sigma_{2}\right)\left(\gamma s+\sigma_{3}\right) \cdots\left(\gamma s+\sigma_{n}\right)
$$

$k_{1}=-\sigma_{1} \sigma_{2} \sigma_{3} \cdots \sigma_{n}$

$k_{2}=-\left(\sigma_{1} \sigma_{2} \sigma_{3} \cdots \sigma_{n-1}+\sigma_{1} \sigma_{3} \sigma_{4} \cdots \sigma_{n}+\cdots+\sigma_{2} \sigma_{3} \sigma_{4} \cdots \sigma_{n}\right)$

$k_{3}=-\left(\sigma_{1} \sigma_{2} \sigma_{3} \cdots \sigma_{n-2}+\sigma_{1} \sigma_{3} \sigma_{4} \cdots \sigma_{n-1}+\cdots+\sigma_{3} \sigma_{4} \cdots \sigma_{n}\right)$

$k_{n}=-\left(\sigma_{1}+\sigma_{2}+\cdots+\sigma_{n}\right)$

이득조절 요소가 추가된 제어기의 수렴속도에 관한 최종 출력 식은 식 (31)과 같다.

$$
y(t)=N_{1} e^{-\frac{\sigma_{1}}{\gamma}}+N_{2} e^{-\frac{\sigma_{2}}{\gamma}}+\cdots+N_{n} e^{-\frac{\sigma_{n}}{\gamma}}
$$

한편, 보상기가 추가된 $n$ 차 상태 궤환 제어기에서 최종 수 렴범위와 수렴속도를 결정하는 라플라스 식은 아래와 같다.

$X_{1}(s)=$

$\frac{k_{i} \alpha_{i} \omega_{i} s}{\underbrace{\left(s^{2}+\omega^{2}\right)}_{\text {최종 수렴범위 }} \underbrace{\left(\beta s^{n+1}+\left(1-k_{n} \beta\right) s^{n}-\left(k_{n}+k_{n-1} \beta\right) s^{n-1}-\cdots-k_{1} \beta s-k_{1}\right)}_{\otimes \otimes}}$

$\otimes \otimes$ 은 수렴속도를 결정한다. 수렴속도와 관련된 식을 인수 분해 하여 정리하면 식 (33)와 같다.

$$
\begin{aligned}
& \otimes \otimes=\left(\beta s+\bar{\sigma}_{1}\right)\left(s+\bar{\sigma}_{2}\right) \cdots\left(s+\bar{\sigma}_{n}\right)\left(s+\bar{\sigma}_{n+1}\right) \\
& k_{1}=-\bar{\sigma}_{1} \bar{\sigma}_{2} \cdots \bar{\sigma}_{n} \bar{\sigma}_{n+1} \\
& k_{2}=-\beta k_{1}-\left(\bar{\sigma}_{1} \bar{\sigma}_{2} \cdots \bar{\sigma}_{n}+\bar{\sigma}_{1} \bar{\sigma}_{3} \bar{\sigma}_{4} \cdots \bar{\sigma}_{n+1}+\cdots+\beta \bar{\sigma}_{2} \bar{\sigma}_{3} \bar{\sigma}_{4} \cdots \bar{\sigma}_{n+1}\right) \\
& k_{3}=-\beta k_{2}-\left(\bar{\sigma}_{1} \bar{\sigma}_{2} \bar{\sigma}_{3} \cdots \bar{\sigma}_{n-1}+\bar{\sigma}_{1} \bar{\sigma}_{3} \bar{\sigma}_{4} \cdots \bar{\sigma}_{n}+\cdots+\beta \bar{\sigma}_{3} \bar{\sigma}_{4} \cdots \bar{\sigma}_{n+1}\right) \\
& \quad \vdots \\
& k_{n}=\frac{1}{\beta}\left(1-\left(\bar{\sigma}_{1}+\beta \bar{\sigma}_{2}+\cdots+\beta \bar{\sigma}_{n+1}\right)\right)
\end{aligned}
$$

보상기가 추가된 제어기의 수렴속도에 관한 최종 출력 식 은 식 (35)와 같다.

$$
y(t)=M_{1} e^{-\frac{\bar{\sigma}_{1}}{\beta}}+\cdots+M_{n} e^{-\bar{\sigma}_{n}}+M_{n+1} e^{-\bar{\sigma}_{n+1}}
$$

식 (31)과 (35)에서 $N=\left[N_{1}, N_{2}, \cdots, N_{n}\right], \quad M=\left[M_{1}, \cdots, M_{n}\right.$, $M_{n+1}$ ] 은 출력 식을 계산했을 때 각각의 상수 계수이다. 지 수의 $\gamma, \beta$ 값이 커질수록 지수의 전체 크기는 작아져 수렴 속도가 늦어진다. 결론적으로 $\gamma, \beta$ 값이 커지면 최종 수렴범 위는 감소하지만 수렴속도는 늦어진다. 이러한 문제점을 다 음 장에서 스위칭 제어 기법을 통해 최종 수렴범위를 감소시 키고 늦어지는 수렴속도를 줄이는 방법 제시한다.

2. 스위칭 제어 기법이 추가된 제어기

최종 수렴범위의 크기는 과도상태(transient state)보다 정상 상태(steady state)에서 중요한 부분이다. 과도상태 $\left(t_{0} \leq t<t_{s}\right.$ (time of switching)) 에서는 상대적으로 작은 $\gamma_{1}, \beta_{1}$ 값으로 수렴속도를 줄이고, 정상상태 $\left(t_{s} \leq t\right)$ 에 들어가는 기점에는 상대적으로 큰 $\gamma_{2}, \beta_{2}$ 값으로 스위칭 제어 함으로써 최종 수 렴범위를 줄이는 방법을 적용한다.

<초기조건>

i) $\gamma_{2}>\gamma_{1} \geq 1, \quad\left(\beta_{2}>\beta_{1} \geq 1\right)$

ii) $t_{s}>t_{0}=0$

<스위칭 논리식>

if $t_{0} \leq t<t_{s} \rightarrow \gamma=\gamma_{1},\left(\beta=\beta_{1}\right)$

else $t_{s} \leq \mathrm{t} \rightarrow \gamma=\gamma_{2},\left(\beta=\beta_{2}\right)$

$<t_{s}$ 의 디자인 규칙>

$t_{s}$ 는 $t_{0}<t_{s} \leq t_{p}$ 사이의 구간이다. 여기서 $t_{0}$ 는 0 초 이고, $t_{p}$ 는 $\gamma_{1}, \beta_{1}$ 을 적용했을 때 변곡점(point of inflection)이 발생 하는 시간을 말한다. 변곡점의 시간을 기점 $\left(t_{p}\right)$ 으로 $t_{s}$ 의 구 간을 $t_{0}<t_{s} \leq t_{p}$ 와 같이 표현한 이유는 $t_{p}$ 이전의 시간에서 $\gamma_{1}, \beta_{1}$ 와 $\gamma_{2}, \beta_{2}$ 의 출력 값이 유사하기 때문이다. 그래프 상 에서 $\gamma_{1}, \beta_{1}$ 을 적용하여 시뮬레이션 했을 때 시각적으로 기 울기가 0 이 되는 시간을 확인 할 수 있다. 그 시간을 근방으 로 가장 수렴속도를 개선하는 스위칭 시간을 시뮬레이션을 통해 찾아주어야 한다. 이와 같이 $t_{s}$ 를 디자인 한다면 스위칭 제어를 했을 때 과도상태에서 효과적으로 수렴속도를 줄일 수 있고 정상상태에서는 $\mathrm{AC}$ 측정 노이즈의 크기를 $10 \%$ 이하 로 줄일 수 있다.

\section{VI. 시뮬레이션}

제안된 제어기법을 검증하기 위해 4차 시스템에서 $x_{1}(t)$ 에 측정 노이즈가 포함된 경우와, 3 차 시스템에서 $x_{2}(t)$ 에 측정 에러가 포함된 경우를 시뮬레이션 하였다.

Case 1: $x_{1}(t)$ 에 측정 노이즈가 포함된 4차 시스템으로 초 기값은 $x_{1}(0)=1, x_{2}(0)=-2, x_{3}(0)=3, x_{4}(0)=-4$, 제어기 이득값은 $K=[-840,-638-179-22], \mathrm{DC}$ 측정 노이즈는 $d_{1}=7, \mathrm{AC}$ 측정 노이즈는 $\alpha_{1} \sin \omega_{1} t=0.6 \sin 2 t$ 로 설정한다. 


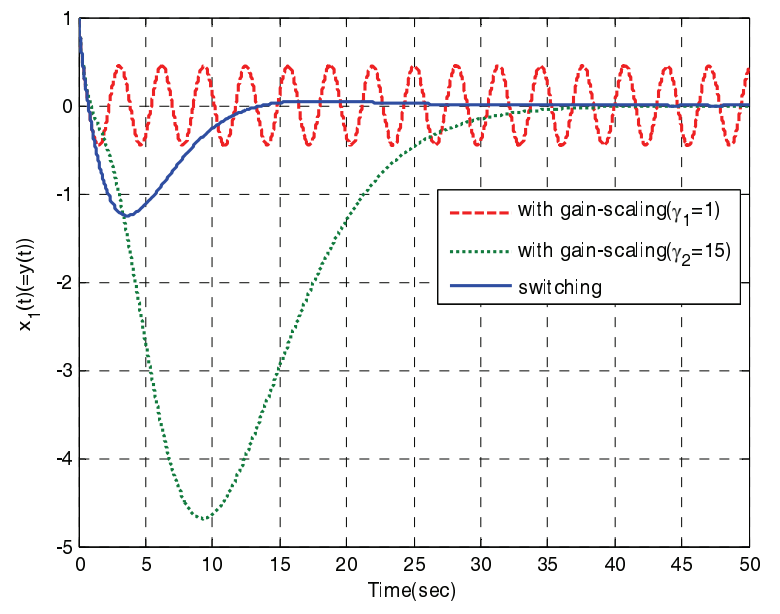

그림 1. $x_{1}(t)$ 에 측정에러가 포함된 4차 시스템

Fig. $1.4^{\text {th }}$ order system including measurement error of $x_{1}(t)$.

그림 1 은 $\gamma_{1}=1, \gamma_{2}=15$ 그리고 스위칭 제어의 시뮬레이션 결과이다. 이득조절 요소가 포함된 제어기의 스위칭 논리식 은 아래와 같다.

$$
\begin{aligned}
& \text { if } \quad 0 \leq t<0.617, \quad \gamma=\gamma_{1} \\
& \text { else } \quad 0.617 \leq t, \quad \gamma=\gamma_{2}
\end{aligned}
$$

$\mathrm{DC}$ 측정 노이즈는 미분으로 인해 값이 사라진다. $\mathrm{AC}$ 측정 노이즈의 크기는 0.6 이고 스위칭 제어 기법을 적용했을 때 최종 수렴범위의 크기는 0.001 이다. 결과적으로 스위칭 제어 을 함으로써 효과적으로 수렴속도를 줄였고 최종 수렴범위 의 크기는 $\mathrm{AC}$ 측정 노이즈의 크기에 $1 \%$ 도 되지 않는다.

Case 2: $x_{2}(t)$ 에 측정 노이즈가 포함된 3차 시스템으로 초 기값은 $x_{1}(0)=1, x_{2}(0)=-2, x_{3}(0)=3$, 제어기 이득값은 $K=$ $[-6000,-1100,-60], \mathrm{DC}$ 측정 노이즈는 $d_{2}=5, \mathrm{AC}$ 측정 노이즈는 $\alpha_{2} \sin \omega_{2} t=2 \sin 2 t$ 로 설정한다.

그림 2는 보상기가 없을 때, 보상기가 있을 때 그리고 스 위칭 제어의 시뮬레이션 결과이다. 보상기가 포함된 제어기 의 스위칭 논리식은 아래와 같다.

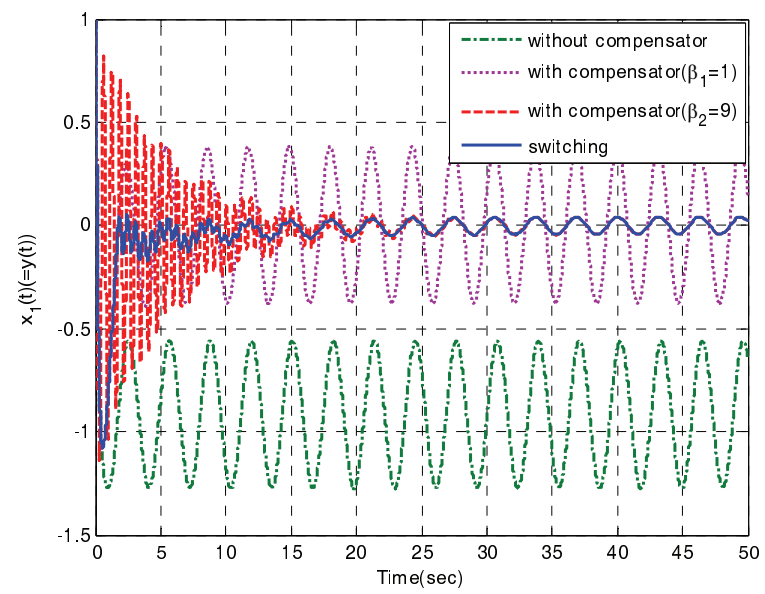

그림 2. $x_{2}(t)$ 에 측정에러가 포함된 3 차 시스템.

Fig. 2. $3^{\text {rd }}$ order system including measurement error of $x_{2}(t)$.

$$
\begin{aligned}
& \text { if } \quad 0 \leq t<1.6, \quad \beta=\beta_{1} \\
& \text { else } \quad 1.6 \leq t, \quad \beta=\beta_{2}
\end{aligned}
$$

$\mathrm{DC}$ 측정 노이즈는 보상기로 인해 값이 사라진다. $\mathrm{AC}$ 측정 노이즈의 크기는 2이고 스위칭 제어 기법을 적용했을 때 최 종 수렴범위의 크기는 0.04 이다. 따라서 최종 수렴범위의 크 기는 $\mathrm{AC}$ 측정 노이즈의 크기에 $2 \%$ 밖에 되지 않는다.

\section{VII. 결론}

본 논문에서는 센서의 오작동으로 상태변수 $x_{i}(t)$ 에 미지 의 정현파 형태의 $\mathrm{AC}$ 측정 노이즈와 알 수 없는 $\mathrm{DC}$ 측정 노이즈가 포함되었음을 가정하였다. 상태변수 $x_{1}(t)$ 에 측정 노이즈가 포함되었을 경우 제어기에 이득조절 요소를 추가 하였고, $x_{1}(t)$ 을 제외한 상태변수 $x_{i}(t)$ 에 측정 노이즈가 포 함되었을 경우엔 보상기를 추가하여 설계하였다. 이득조절 요소인 $\gamma$ 값과 보상기 상수인 $\beta$ 를 증가 시킴으로써 효과적 으로 최종 수렴범위의 크기를 줄일 수 있었다. 또한 $\gamma$ 값과 $\beta$ 값이 커짐에 따라 늦어지는 수렴속도를 줄이기 위해 스위 칭 제어 기법을 적용하여 수렴속도를 감소시킬 수 있었다. 측정 노이즈의 영향으로 발생하는 최종 수렴범위의 크기와 이득조절 요소 그리고 최종 수렴범위의 크기와 보상기의 관 계를 라플라스 변환 기법을 통하여 해석하였고, 4차 시스템 의 경우와 3차 시스템의 경우를 MATLAB 시뮬링크를 통해 시뮬레이션 하여 제어기의 유효성을 검증하였다.

\section{REFERENCES}

[1] C.-T. Chen, Linear System Theory and Design, 3rd Ed., Oxford University Press Inc., 1999.

[2] Z. Chen, "A remark on sensor disturbance rejection of nonlinear systems," IEEE Transactions on Automatic. Control, vol. 54, no. 9, pp. 2206-2210, 2009.

[3] R. A. Freeman, "Global internal stabilization does not imply global external stabilizability for small sensor disturbances," IEEE Trans. Automat. Control, vol. 40, no. 12, pp. 2119-2122, Dec. 1995.

[4] S. Gayaka and B. Yao, "Global stabilization of a chain of integrators with input saturation and disturbances," American control conference, O'Farrell Street, San Francisco, CA, USA, Jun.-Jul. 2011.

[5] H.-D. Kim and H.-L. Choi, "An output feedback controller for a ball and beam system under measurement noise of feedback sensor," Journal of Institute of Control, Robotics and Systems (in Korean), vol. 17, no. 10, pp. 955-959, 2010.

[6] H.-D. Kim and H.-L. Choi, "Design and analysis of an output feedback controller for a chain of integrators system compensating measurement noise of feedback sensor," Journal of Institute of Control, Robotics and Systems (in Korean), vol. 17, no. 4, pp. 299-303, Nov. 2011.

[7] H.-D. Kim and H.-L. Choi, "Measurement output feedback control with a switching gain-scaling factor for a chain of integrators under sensor noise," IEICE Trans. Fundamentals, vol. E95-A, no. 9, pp. 1623-1626, Sep. 2012.

[8] H. K. Khalil, Nonlinear Systems, 3rd Ed., Prentice Hall Inc., 2002. 
[9] A. Serrani, A. Isidori, and L. Marconi, "Semi-global nonlinear output regulation with adaptive internal model," IEEE Trans. Autom. Control, vol. 46, no. 8, pp. 1178-1194, 2001.

[10] D.-H. Yeom and J.-Y. Choi, "Switching control for second order nonlinear systems using singular hyperplanes," International Journal of Control, Automation, and System, vol. 4, no. 1, pp. 124-135, Feb. 2006

[11] J.-S. Youn, H.-D. Kim, and H.-L. Choi, "Control of a chain of integrators with a delay in the input under measurement feedback," IEICE Trans. Fundamentals, vol. E94-A, no. 6, pp. 1464-1467, Jun. 2011.

[12] J.-S. Youn and H.-L. Choi, "Design and analysis of a state feedback controller for a chain of integrators system under measurement noise," Journal of Institute of Control, Robotics and Systems (in Korean), vol. 16, no. 10, pp. 969-974, 2010.

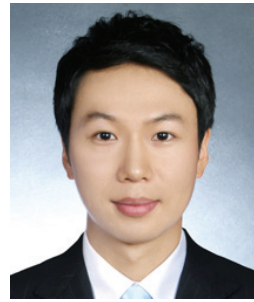

최 호 림

\section{오 상 영}

2013년 동아대학교 전기공학과 졸업. 2013년 현재 동아대학교 대학원 전기공 학과 석사과정 재학중. 관심분야는 제 어이론, 비선형 시스템 제어.

제어 · 로봇 - 시스템학회 논문지 제 15 권 제 4 호 참조. 